\title{
Stress Calculation of Polymer Displacing Residual Oil in Micro Pores
}

\author{
LILI LIU*, SHUREN YANG and JIAWEI FAN \\ Key Laboratory of Enhanced Oil and Gas Recovery of Education Ministry at Northeast \\ Petroleum University, Daqing163318, Heilongjiang, China
}

(Received on September 03, 2016, revised on October 16, 2016)

\begin{abstract}
To analyze and calculate the micro-scale stress of viscoelastic fluid in displacing residual oil and explore the rheological property of non-Newtonian fluid from hydrodynamics angle, an upper-convected Maxwell constitutive equation was selected to simulate viscoelastic fluid. Furthermore, boundary conditions were adopted to calculate the flow field of flow, and the normal deviatoric stress and horizontal stress difference acting on residual oil from viscoelastic fluid were calculated by combining stress tensor theory. The calculated result showed that: the viscosity, elasticity and flow rate of polymer solution are key factors influencing stress and deformation of residual oil. Therefore, methods like increasing mass concentration and molecular mass of polymer solution can be applied to enlarge viscosity and elasticity of polymer solution, thus increasing the displacement stress acting on residual oil and laying foundation for further analysis on deformation and breakup of residual oil.
\end{abstract}

Key words: Polymer flooding, Normal deviatoric Stress, Horizontal stress difference, Micro pore

\section{Introduction}

From the macro perspective, visco-elastic polymer solution flooding oil is used in high water cut oilfields. The polymer solution has high viscosity, which can effectively improve the oil and water mobility ratio, ease the contradiction between the layers, improve the liquid suction profile, which can effectively enlarge sweep volume of displacement fluid. From the micro perspective, because the polymer solution has a degree of elasticity that can increase the displacement force on residual oil, the displacement efficiency is further improved. In enhanced oil recovery, polymer solution can enlarge swept volume and enhance oil displacement efficiency.

Yin Hongjun[1-2], et al adopted the corrected upper-convected Maxwell constitutive model to show that using numerical simulation, the larger the elasticity of viscoelastic fluid, the larger the sweep efficiency. Xia Huifen[3-5], Wang Demin, et al proposed that viscoelastic fluid can enhance oil displacement efficiency, made a detailed qualitative analysis on micro-scale stress acting on residual oil to point out that micro-scale stress in micro pore space makes residual oil deformed and further separated, and verified the mechanism of action of polymer solution on residual oil experimentally. Ryan R.wilton and Farshid Torabi[6] studied the difference between hydrophobically associating amphiphilic polymer (HAP) and conventional partially hydrolyzed polyacrylamide solution (HPAM), focused on the mechanism of HAP in enhancing the elasticity of polymer solution, and analyzed the advantages of HAP in heavy oil recovery. Qualitative analysis [7-10] made in experiment shows that the viscoelastic property of polymer can

*Corresponding author's email: dqliul12009@163.com 
enhance recovery efficiency, but qualitative calculation on the stress of polymer during oil displacement process acting on residual oil, the relationship between displacement stress and elasticity of polymer were not conducted.

In the paper, a research was carried out, aimed at stress of residual oil adhered on wall surface in micro pores in flow field of viscoelastic polymer solution. Upper-convected Maxwell constitutive equation was selected to simulate viscoelastic polymer solution, normal deviatoric stress and horizontal stress difference acting on residual oil were calculated, and comparison was made on rheological property of viscoelastic polymer solution and flow path's geometric parameters on residual oil stress, finally laying foundation for analysis on deformation and breakup of residual oil.

\section{Simplification of Model}

Irrespective of complicated internal spatial structure of porous media, flow path was simplified as an uni-diameter capillary tube, and accordingly residual oil was located at the bottom of capillary tube. An analysis was made on stress acting on residual oil from viscoelastic polymer solution in micro pores when flowing through residual oil under action of differential pressure. A physical model of residual oil is shown in Figure 1.

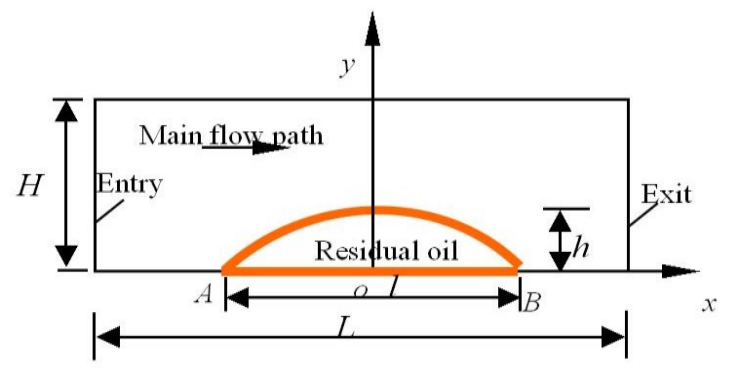

Fig. 1: Physical model

It is suitable to adopt upper-convected Maxwell constitutive equation to describe the rheological property of viscoelastic fluid via practice research.

\section{Numerical Calculation of Deviatoric Stress of Viscoelastic Polymer Solution Acting on Residual Oil}

According to the need of research, the stress on the surface of residual oil film is decomposed as shown below, namely decomposition along normal/tangential direction and horizontal/vertical direction, as shown in Fig. 2.

In Figure 2, $\tau_{n \tau}, \tau_{n n}, \tau_{n h}$ and $\tau_{n v}$ respectively refer to tangential deviatoric stress, normal deviatoric stress, horizontal deviatoric stress and vertical deviatoric stress.

According to tensor theory, stress tensor $P$ of any dot on the surface of residual oil film means the stress state of a dot and the stress of this dot with $n$ as normal direction can be expressed as:

$$
\boldsymbol{p}_{\boldsymbol{n}}=\boldsymbol{P} \cdot \boldsymbol{n}
$$

Dot product was made by stress tensor $P$ with respect to unit vector along tangential, normal, vertical and horizontal direction at this dot to gain the tangential deviatoric stress 
$\tau_{n \tau}$, normal deviatoric stress $\tau_{n n}$, vertical deviatoric stress $\tau_{n v}$ and horizontal deviatoric stress $\tau_{n h}$ at this dot.
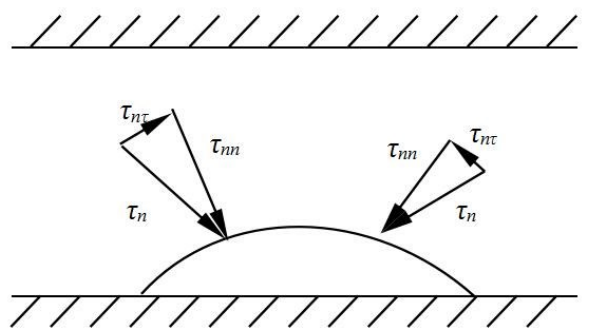

(a) Normal and tangential decomposition
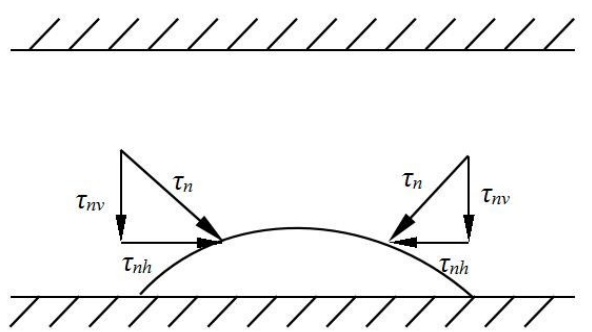

(b) Horizontal and vertical decomposition

Fig. 2: Two Types of Decomposition Diagram of Oil Film Stress

Calculation was respectively made on the change of deviatoric stress acting on residual oil film from factors such as viscosity and elasticity of displacing fluid, width of flow path, flow rate. In this paper, positive and negative rules on normal deviatoric stress cause pressure to be positive and tension to be negative while these rules on tangential stress cause flow direction to be positive and the contrary one to be negative.

\section{1. Calculation of Normal Deviatoric Stress}

Suppose residual oil stays still, oil displacement process of polymer solution belongs to steady flow. Basic parameters: width of flow path: $20 \mu \mathrm{m}$; length: $100 \mu \mathrm{m}$; length of residual oil film: $40 \mu \mathrm{m}$; height: $10 \mu \mathrm{m}$. Calculation conditions were shown in Table 1. Weissenberg number $W e$ is a factor reflecting the elasticity of fluid, and its calculation formula is

$$
W e=\lambda \frac{u}{l}
$$

In the formula: We refers to Weissenberg number; $\lambda$ refers to relaxation time; $u$ refers to characteristic velocity; $l$ refers to characteristic length. We is directly proportional to relaxation time. A larger We causes a stronger elasticity of fluid. Polymer solution is viscoelastic fluid, so evolution was adopted during the process of calculation to conduct gradual processing for elasticity item.

Table 1: Calculation Conditions

\begin{tabular}{|c|c|c|c|c|c|}
\hline $\begin{array}{c}\text { Flow rate } \\
Q /\left(\mathrm{m}^{3} / \mathrm{s}\right)\end{array}$ & $\begin{array}{c}\text { Zero-shear viscosity of } \\
\text { viscoelastic fluid } \eta_{p} /(\mathrm{mPa} \cdot \mathrm{s})\end{array}$ & $\begin{array}{c}\text { Viscosity of residual } \\
\text { oil film } \eta_{o} /(\mathrm{mPa} \cdot \mathrm{s})\end{array}$ & $W e$ & $R e$ & $\begin{array}{c}\text { Initial wetting } \\
\text { angle } \theta /\left({ }^{\circ}\right)\end{array}$ \\
\hline $2 \mathrm{e}-10$ & 30 & 20 & 0.2 & $7 \mathrm{e}-6$ & 47 \\
\hline
\end{tabular}

Change curve of normal deviatoric stress under different conditions is given in Fig.3. 


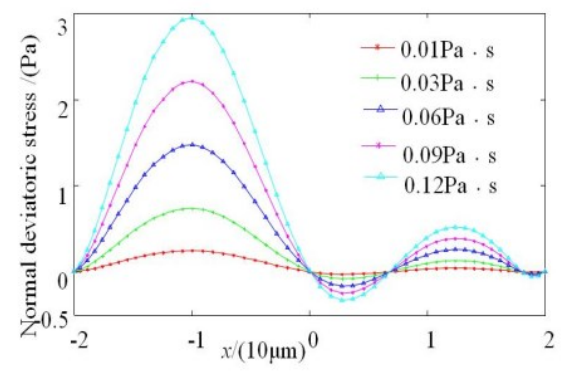

(a) Viscosity change

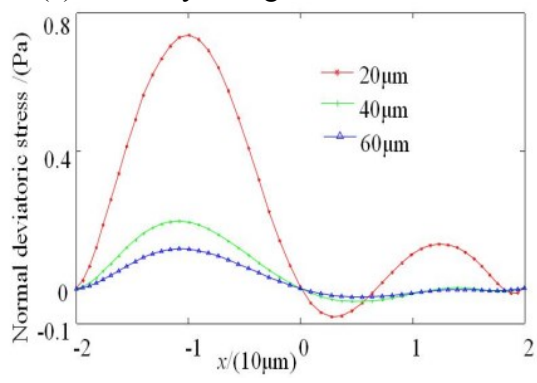

(c) Change of flow path width

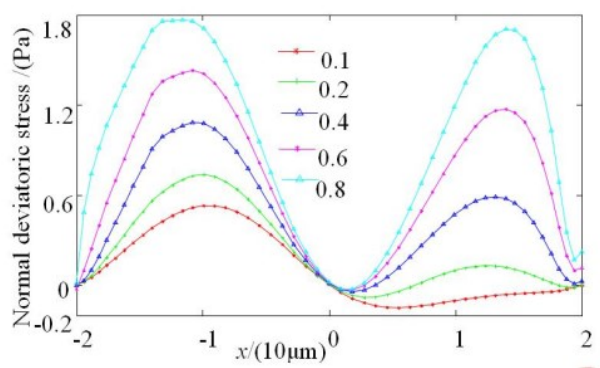

(b) We change

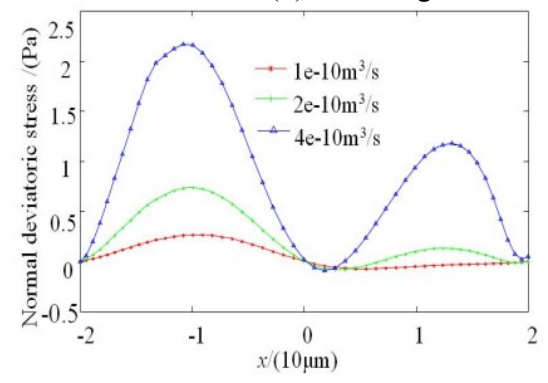

(d) Change of flow rate

Fig. 3: Change Curve of Normal Deviatoric Stress

Normal deviatoric stress is a force vertical to residual oil surface, which leads to normal heave or concave of residual oil. This is beneficial to enlarging local deformation of residual oil. Factors such as physical property parameters of displacing fluid, size of flow path and the characteristics of residual oil affect normal deviatoric stress. It can be seen from Figure 3 that the smaller the viscosity ratio between residual oil and polymer solution, the larger the viscosity of polymer solution and normal devistoric stress acting on residual oil film; under the same flow rate, a larger width of flow path indicates a larger area of cross section of passage, and if the flow velocity reduces, the force acting on residual oil film will reduce; if the flow rate increases, the force acting on residual oil will increase.

Change trend of normal deviatoric stress is similar in above conditions, normal deviatoric stress gradually increases in the upstream of oil film, but gradually decreases after reaching the extreme value; oil film is pressured, and normal deviatoric stress is negative near the middle of oil film, indicating that oil film is tensioned. Then the normal deviatoric stress acting on oil film is positive, but oil film is still pressured, thus forming pressure at both ends. Distribution of tension in the middle helps oil film to be bulged, then deformed under the constant action of displacing fluid, and finally separated from the ontology of oil film.

\section{2. Calculation of Tangential Deviatoric Stress}

Change curve of tangential deviatoric stress is shown in Figure 4. 


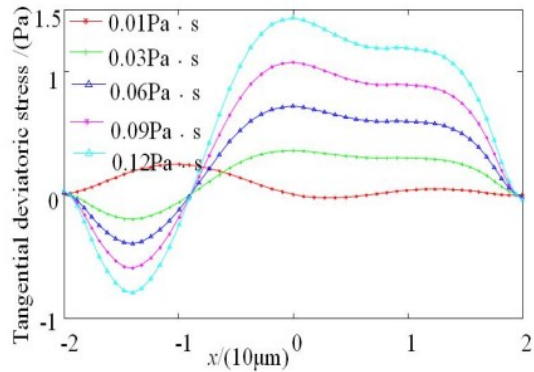

(a) Viscosity change

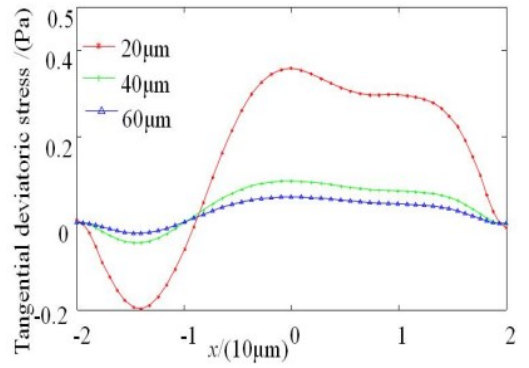

(c) Change of flow path width

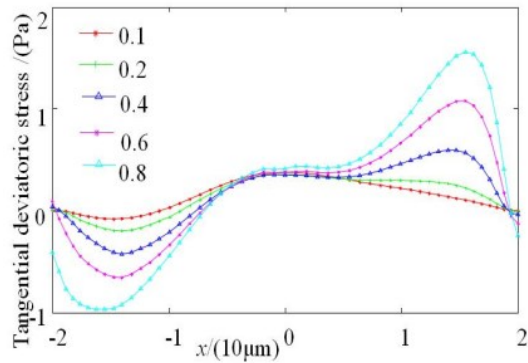

(b) We change

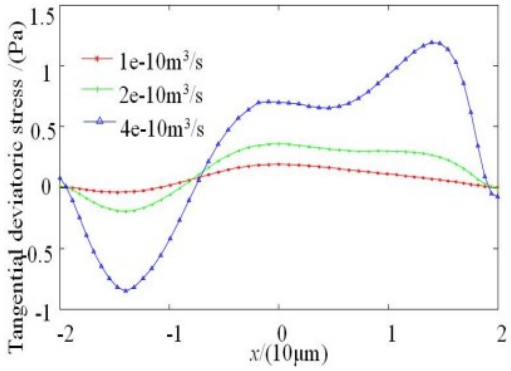

(d) Change of flow rate

Fig. 4: Change Curve of Tangential Deviatoric Stress

Tangential deviatoric stress is a force along the tangential direction of residual oil film, the larger the force, the larger the angular deformation. There is a demarcation point between $1 / 4$ and $1 / 2$ of oil film along the flow direction, before and behind which the tangential deviatoric stress is separately negative and positive. Factors such as physical property parameters of displacing fluid, size of flow path and the characteristics of residual oil affect tangential deviatoric stress. The smaller the viscosity ratio between residual oil and displacing fluid, the stronger the elasticity and the larger the tangential deviatoric stress acting on residual oil. Viscoelastic fluid has memory function, so the elasticity of displacing fluid produces a lag influence on peak value of tangential deviatoric stress. The wider the flow path, the smaller the flow rate and the smaller the tangential deviatoric stress acts on residual oil.

\section{Numerical Calculation of Horizontal Stress Difference of Viscoelastic Polymer Solution Acting on Residual Oil}

The calculated result of deviatoric stress above can only show the distribution difference of stress acting on residual oil under different parameters, but can't intuitively show the difference of displacement stress acting on residual oil. According to the theory by the academician Wang Demin, the force capable of truly reflecting displacement action of displacing fluid on residual oil should be the difference value of horizontal stress acting on the same horizontal line of residual oil interface- horizontal stress difference. Change curve of horizontal stress difference under different conditions is given in Fig. 5. 


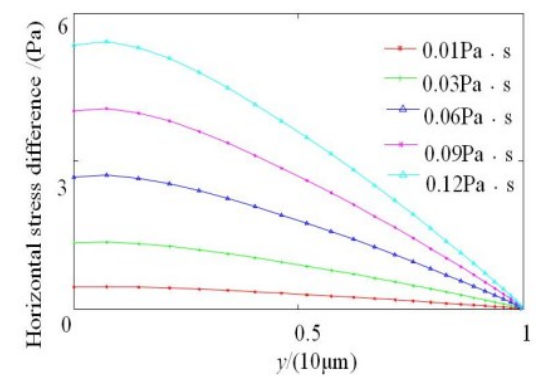

(a) Viscosity change

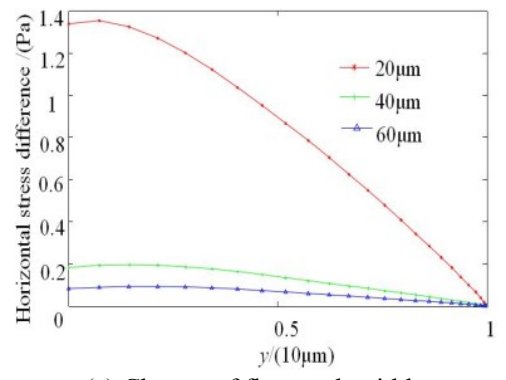

(c) Change of flow path width

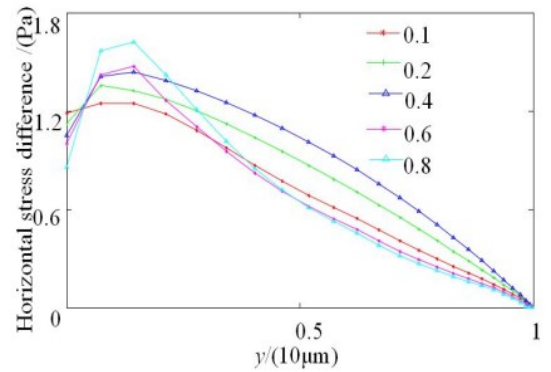

(b) We change

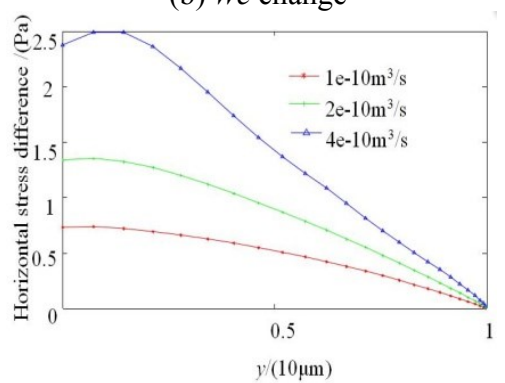

(d) Change of flow rate

Fig. 5: Change Curve of Horizontal Stress Difference

Horizontal stress difference reflects the size of residual oil displacement stress at different heights along the flow direction. Fig. 5 shows that horizontal stress difference will increase if the viscosity of displacing fluid increases, and if the viscosity doubles, so does the horizontal stress difference. The distribution of horizontal stress difference changes with an increase of the elasticity of displacing fluid, and if the elasticity of displacing fluid is larger, the horizontal stress difference will increase to the extreme value from a smaller one, then decrease gradually, namely the extreme point is not a three-phase junction but is located at certain position of the upstream of residual oil film, which is also relevant to the memory function of viscoelastic fluid, the larger the elasticity of displacing fluid, the larger the extreme value of horizontal stress difference. A larger width of flow path causes a smaller horizontal stress difference. If the ratio of flow path width is $1: 2: 3$, the extreme value ratio of horizontal stress difference is 13.5:2:1. This result is just contrary to that under the condition of constant differential pressure; the larger the flow rate, the larger the horizontal stress difference. If the flow rate ratio is $1: 2: 4$, the extreme value ratio of horizontal stress difference is 1:1.8:3.3.

Because of restriction of calculation conditions, this paper was only calculated to 0.8 ; when the elasticity of polymer displacing fluid increases and We exceeds 0.8 , the change trend of horizontal stress difference can only be judged by drawing the max. value of horizontal stress difference. See Fig. 6.

It can be seen from Fig. 6 that if other conditions are kept unchanged, the extreme value of horizontal stress difference progressively increases in quadratic function as $\mathrm{We}$ increases, which means the larger the elasticity of polymer solution, the larger the max. value of horizontal stress difference, the larger the increase range. 


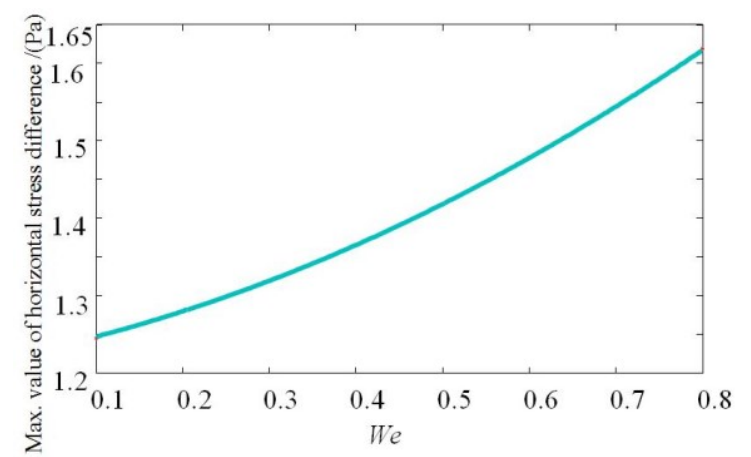

Fig. 6: Relation Curve between Max. Value of Horizontal Stress Difference and We

\section{Indoor Rheological Property Experiment}

By conducting indoor polymer solution rheological testing experiment, an analysis was made on the influence of mass concentration of polymer solution and the molecular mass of polymer upon the viscoelastic property of polymer solution. RS-150 type rheometer produced by German HAAKE Company was applied to carry out vibration experiment for polymer solution, and $\mathrm{C} 60 / 1 \mathrm{Ti}$ cone plate system (radius of cone plate is $60 \mathrm{~mm}$ and the conical degree is $1^{\circ}$ ) was applied for dynamic shear test. The polymer for experimental use is common anionic HPAM produced by Daqing Refining \& Chemical Company, and the relative molecular mass is respectively 15 million, 25 million and 35 million. Stir for $120 \mathrm{~min}$ with stirrer to prepare polymer solution in various concentrations, keep still for $30 \mathrm{~min}$ to ensure intensive mixing. Water for experimental use is distilled water, simulated salt water is artificially prepared, and the degree of mineralization is $508 \mathrm{mg} / \mathrm{L}$. The computer automatically controls the test, processes data and draws diagrams. The temperature is constant temperature of $45 \square$, and the test frequency is $0.046 \sim 25.100 \mathrm{~Hz}$. Fig. 7 shows the rheological curve of polymer solution in different mass concentrations.

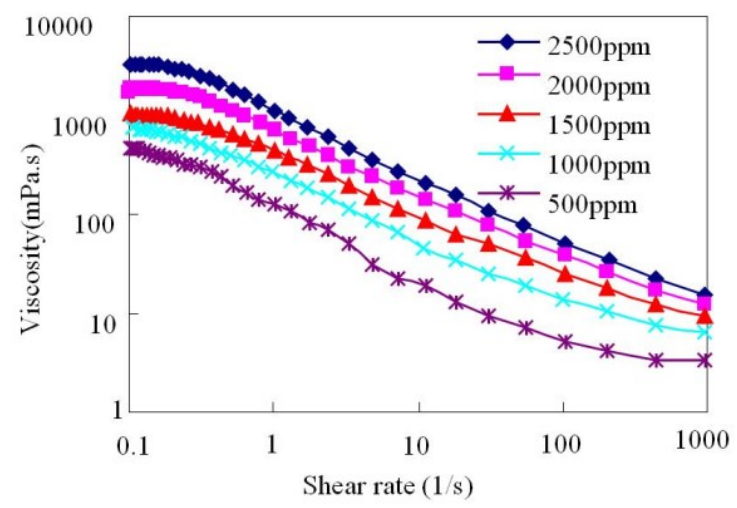

Fig. 7: Viscosity of Polymer Solution in Different Mass Concentrations

The Fig. 7 shows that under double logarithm coordinate, as shear rate increases, 
polymer solution viscosity decreases, and the two are approximately a line relationship. The viscosity increases as the mass concentration of polymer solution increases. Figure 8 (a) and (b) are separately the change curve of storage modulus and loss modulus of polymer solution in different mass concentrations along with angular velocity (relative molecular mass: 15 million; degree of mineralization: $508 \mathrm{mg} / \mathrm{L}$ ).
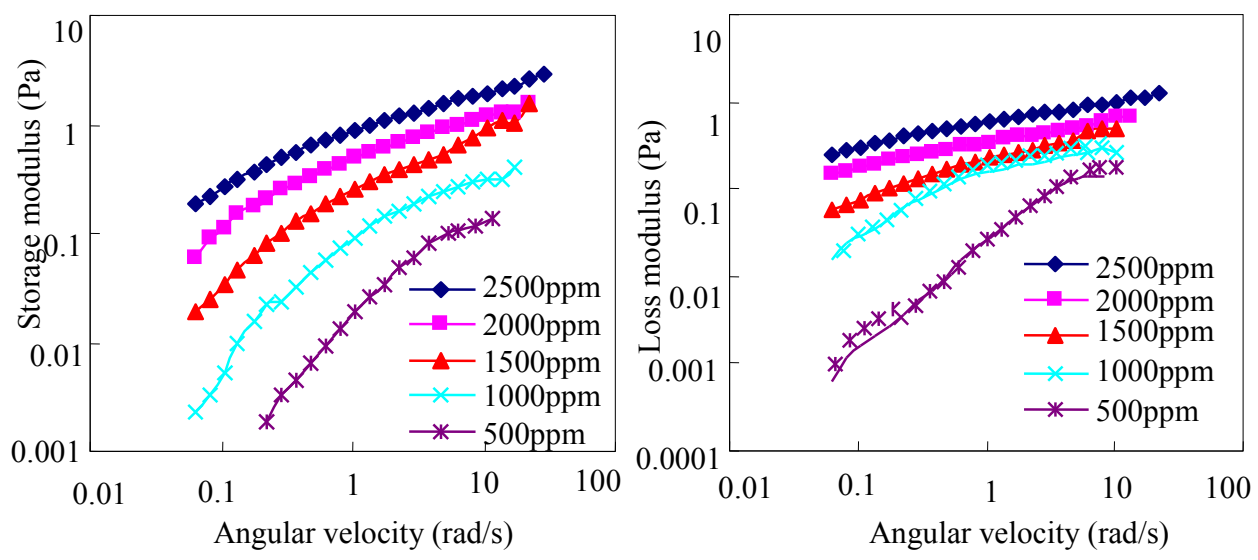

Fig. 8: Storage Modulus and Loss Modulus of Polymer Solution in Different Mass Concentrations

It can be observed from Figure 8 that as angular velocity increases, the storage modulus and loss modulus increase; as the mass concentration of polymer solution increases, the storage modulus and loss modulus increase. This is because as mass concentration of polymer solution increases, the number of molecules in unit volume increases, and the ability of mutual attraction and mutual entanglement among molecules becomes stronger. Figure 9 shows the rheological curve of polymer solution in different relative molecular masses (mass concentration: $1500 \mathrm{mg} / \mathrm{L}$; degree of mineralization: $3700 \mathrm{mg} / \mathrm{L})$.

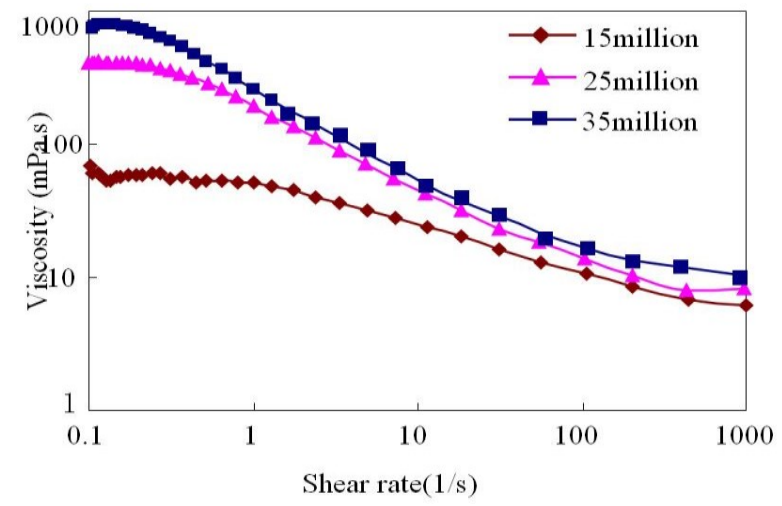

Fig. 9: Viscosity of Polymer Solution in Different Relative Molecular Masses

Fig. 9 shows that as the relative molecular mass of polymer increases, the viscosity increases. Figure 10 (a) and (b) are separately the change curve of storage modulus and loss modulus of polymer solution in different relative molecular masses along with 
angular velocity (mass concentration: $1500 \mathrm{mg} / \mathrm{L}$; degree of mineralization: $3700 \mathrm{mg} / \mathrm{L}$ ).
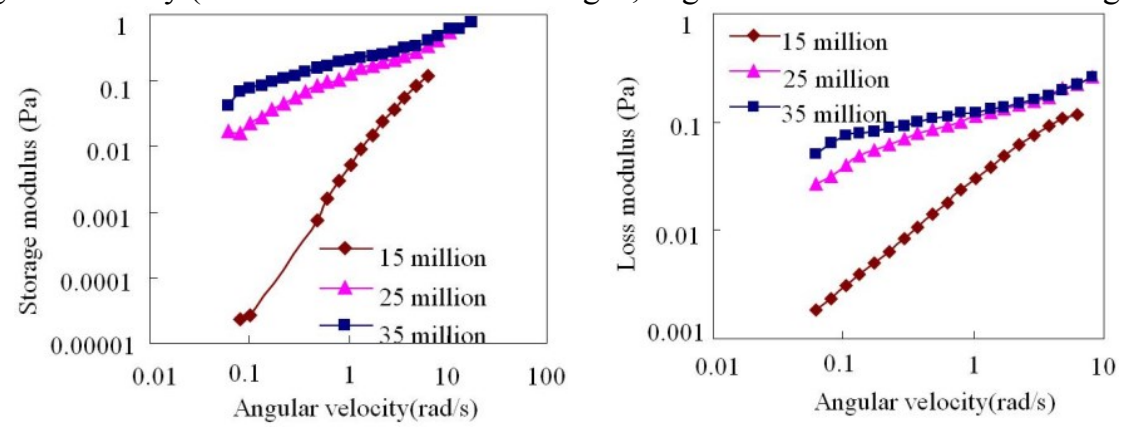

Fig. 10: Storage Modulus and Loss Modulus of Polymer in Different Relative Molecular Masses

It can be observed from Figure 10 that as angular velocity increases, the storage modulus and loss modulus increase; as relative molecular mass of polymer solution increases, the storage modulus and loss modulus increase. This is because the larger the relative molecular mass is, the larger the attraction among molecules, the more stable the conformation of molecular chain and the stronger the viscoelasticity.

\section{Conclusions}

(1) Normal deviatoric stress is the most important factor to enhance oil recovery by polymer displacing fluid. The elasticity of displacing fluid plays the most significant role in influencing residual oil stress; the stronger the elasticity of displacing fluid, the larger the normal deviatoric stress acting on residual oil film; the larger the flow rate, the narrower the flow path, the larger the force acting on residual oil.

(2) The index-horizontal stress difference $\tau_{\mathrm{nh}}$, which can reflect the displacement stress acting on residual oil, is presented. The calculated result shows that horizontal stress difference has an extreme point on the surface of residual oil film; the max. value of horizontal stress difference increases with the increase of the elasticity of displacing fluid, and increases progressively in parabolic rule with increase of $W e$; the max. value of horizontal stress difference increases with the increase of the viscosity of displacing fluid, basically in linear relation.

(3) Indoor visual oil displacement experiment shows that the elasticity of displacing fluid will increase by increasing the concentration of polymer solution or enlarging the molecular mass of polymer. According to calculation on stress and deformation, if the elasticity of displacing fluid increases, the stress of residual oil will increase, which is beneficial to deformation of residual oil and better displacement effect. This is identical to the experiment. Furthermore, the fluid elasticity capable of enlarging the displacement stress acting on residual oil and deformation is the mechanical mechanism to enhance oil recovery by polymer displacing is presented.

\section{Acknowledgement}

This work was supported by the National Science Foundation in China through grant No. 51374076 and the Northeast Petroleum University in China through grant No.NEPUQN2015-1-05. 


\section{References}

[1] Yin H J, Wang D M, Zhong H Y et al. Flow Characteristics of viscoelastic polymer solution in micro-pores. SPE154640, 2012.

[2] Yin Hongjun, Jiang Haimei, Su Yuchi, et al. Flow behavior of viscoelastic polymer solution in the expansion channel. Acta Polymeric Sinica, 2009,10(6): 520-529.

[3] Xia Huifen, Ye Ju and Fan Shun Kong. Effect of elastic behavior of HPAM solutions on Displacement Efficiency on mixed wettability conditions. SPE90234, 2004.

[4] Xia Huifen, Wang Demin, Zhang Jiuran et al. Quantitative description of contribution of elasticity of polymer solution to oil displacement efficiency. Journal of China University of Petroleum (Edition of Natural Science), 2012, 36(4): 166-176.

[5] Xia Hui-fen, Wang Demin, Wang Gang, et al. Elastic behavior of polymer solution to residual oil at dead-end. Acta Petrolei Sinica, 2006, 27(2): 72-76.

[6] Ryan R. wilton, Farshid Torabi. Rheological Assessment of the Elasticity of Polymers for Enhanced Heavy Oil Recovery. SPE 165488, 2013.

[7] Sandiford B B. Laboratory and field studies of water floods using polymer solutions to increase oil recoveries. JPT, 1964,16(8): 911-916.

[8] Pearson J R A, Tardy, P M J. Models for flow of non-Newtonian and complex fluids through porous media. Non-Newtonian Fluid Mech, 2002,10(2): 447-473.

[9] Duda J L, Klus E E. Flow of polymer solutions in porous media: inadequacy of the capillary model. Ind. Eng. Chem. Fundam,1983, 22(3): 299-305.

[10] Mason G, Morrow N R. Capollar behavior of a perfectly wetting liquid in irregular triangular tubes. Colloid Interface Sci,1991,141(1): 262-274.

Liuli Liu is a researcher at Northeast Petroleum University, China. His major research interests include polymer flooding and fluid mechanism. He is invited as a reviewer by the editors of some international journals, such as Open Journal of Applied Sciences, Open Journal of Fluid dynamics. She has published many papers in related journals.

Dr. Shuren Yang is a researcher at Northeast Petroleum University, China. His major research interests include fluid dynamics and enhanced oil recovery. He is invited as a reviewer by the editors of some international journals, such as Journal of Petroleum Science and Engineering, World Journal of Mechanics. He has published many papers in related journals.

Jiawei Fan is a researcher at Northeast Petroleum University, China. His major research interests include rheology of polymer solution and crude oil. He has published many papers in related areas. 